\title{
A longstanding pigmented lesion
}

Sarah Morton, Jim Muir, Daniel Kennedy

\section{CASE}

A woman aged 70 years presented with a longstanding pigmented lesion on her right ear (Figure 1A). Two years earlier, a 2-mm punch biopsy performed at a difference practice showed focal proliferation of melanocytes, but no atypia, with the pathologist concluding 'unstable solar lentigo' (USL). The patient had not noticed any change in the lesion since.

\section{QUESTION 1}

What is the clinical diagnosis?

\section{QUESTION 2}

Why did the original biopsy fail to show the correct diagnosis?

\section{QUESTION 3}

What is this patient's further management?

\section{ANSWER 1}

The most likely diagnosis is either lentigo maligna or lentigo maligna melanoma (LMM). Figure 1A shows a large, irregularly shaped and variably pigmented lesion with erythema extending from the earlobe to antitragus. There were no proximate similar lesions. Although the diagnosis of lentigo maligna is readily made on clinical features alone, dermoscopic features include rhomboid structures, perifollicular pigment asymmetry, annular granular structures and a grey network. These are all well demonstrated in this case (Figure 1B).

Lentigo maligna is a form of melanoma in situ (MIS), presenting as a slowly growing variably pigmented macule in areas of chronic sun exposure. It is most common in the elderly population. ${ }^{1}$ The reported incidence of lentigo maligna at 13.7 per 100,000 person-years is likely to be underestimated. ${ }^{1} \mathrm{LMM}$ is invasive progression into the dermis, estimated to occur in $4.7 \%$ cases of lentigo maligna. ${ }^{1,2}$ USL is a recently described entity that histologically has melanocytic hyperplasia, but lacks melanocyte nesting, nuclear atypia or hyperchromasia, and is believed to be a precursor to the development of lentigo maligna within solar lentigo. ${ }^{3}$ USL clinically presents as an isolated, irregularly pigmented macule arising on background solar damage. ${ }^{3}$ Comparatively, classic solar lentigo is a keratinocytic proliferation with abnormal pigment retention from hyperactive melanocytes, manifesting as benign, uniformly pigmented macules or patches on sun-exposed areas. ${ }^{3}$ Solar lentigo, USL and lentigo maligna/LMM can appear clinically similar.

\section{ANSWER 2}

Possible reasons:

- The lesion was not lentigo maligna at initial biopsy. This is unlikely, given reliable history that the lesion had not changed in two years.

- Sampling error. A single, small punch biopsy of a large lesion may not be representative.
Lentigo maligna lesions histologically can harbour areas of solar lentigo as well as frank melanoma. ${ }^{4}$ As lentigo maligna arises in solar-damaged skin, it can occur with solar lentigo and within fields of melanocytic hyperplasia. ${ }^{3}$ Regardless of biopsy technique, an adequate specimen should be obtained to optimise a representative sample.

In this case, multiple shave biopsies were performed. Broad shave biopsy specimens produce excellent cosmetic results and broad areas of dermal epidermal junction for accurate histological assessment, reducing the risk of sampling error. ${ }^{4}$ These biopsies showed areas of solar lentigo and lentigo maligna (Figures $1 \mathrm{C}$ and 1D), confirming the clinical impression.

\section{ANSWER 3}

Excision margins of 5-10 $\mathrm{mm}$ have been the standard of care for MIS. ${ }^{5}$ Recent studies have shown that in lentigo maligna, subclinical extension may require larger margins to achieve acceptable clearance rates. ${ }^{5,6}$ If occult invasion or margin involvement is present, re-excision is necessary; therefore, the resultant defect should be closed in a way that does not distort the margin.

Mohs micrographic surgery (MMS) is increasingly used for lentigo maligna/ LMM treatment with improved complete clearance rates, healthy tissue conservation and reduced recurrences due to intraoperative margin assessment before closure..$^{4,7}$ Accessibility to, and 


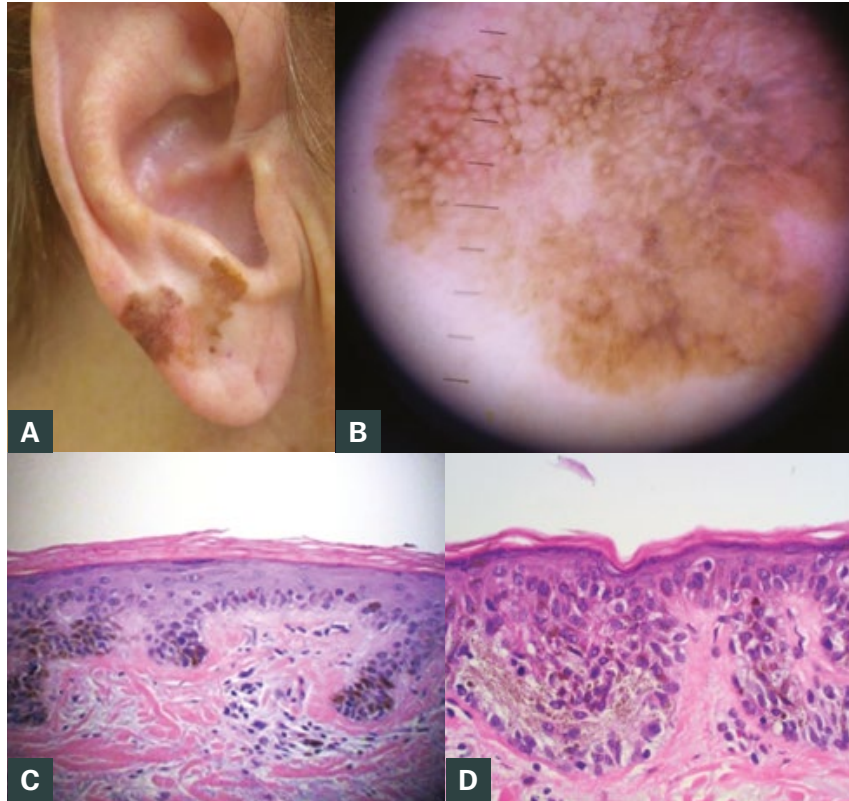

Figure 1. Lesion at presentation

A. Macroscopic appearance; B. Dermoscopy; C. Histopathology demonstrating unstable solar lentigo; D. Histopathology demonstrating lentigo maligna

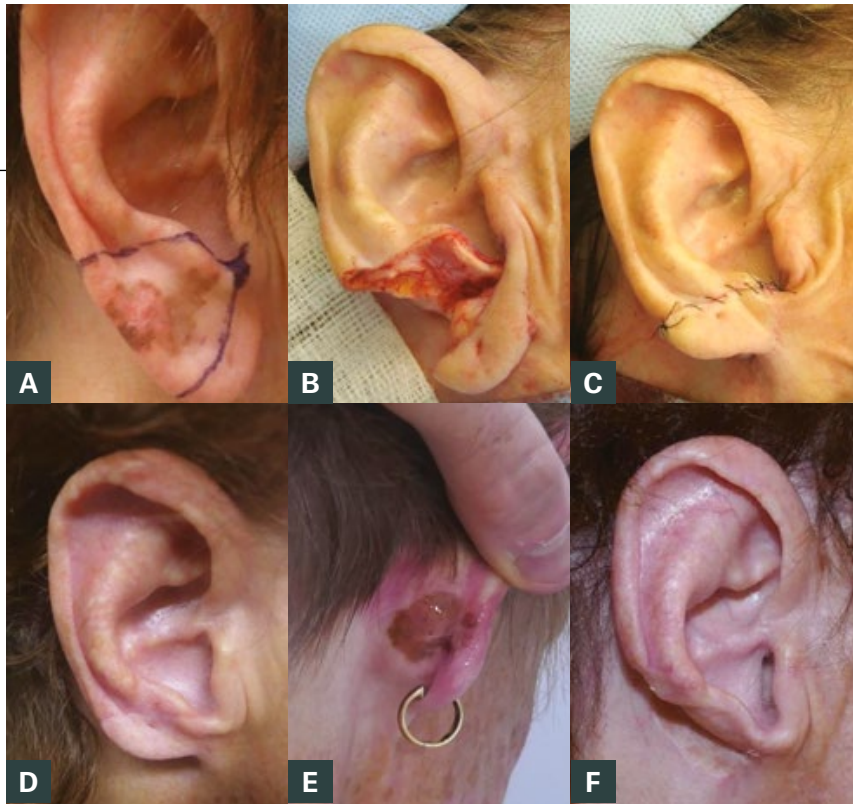

Figure 2. Lentigo maligna management and progression

A. Preoperative excision marked; B. Excision of lesion; C. Closure of wedge excision; D. Six weeks post-operation; E. Recurrence 4.5 years post-initial wedge excision; F. Result of recurrence excision availability of, MMS for lentigo maligna/ LMM patients limits its widespread use.

Regular full skin examination is recommended. There is risk of local recurrence, as well as new primary melanoma and other types of skin malignancy. Education regarding sun protection, warning signs of malignant change and skin self-examination is important. ${ }^{7}$ First-degree relatives should have a full skin examination..$^{5,7}$

\section{CASE CONTINUED}

Subsequent wedge resection was performed by a plastic surgeon (Figures 2A-D). Histopathology confirmed lentigo maligna with clear margins.

The patient had regular full skin examinations without issues until 4.5 years post-excision when she developed a lesion suspected of recurrence at the right posterior ear (Figure 2E). Further excision with skin grafting was performed (Figure 2F). Histopathology confirmed lentigo maligna recurrence with clear margins.
No further evidence of local or systemic recurrence has been seen after two years of regular surveillance. The patient has had keratinocyte malignancies removed.

\section{QUESTION 4}

What is the risk of recurrence?

\section{QUESTION 5}

What are the advantages and potential pitfalls of wedge excision of the ear?

\section{ANSWER 4}

Lentigo maligna has a tendency for subclinical spread, and therefore, high local recurrence rates, despite apparent successful treatment. ${ }^{6,8}$ With conventional excision margins, recurrence rates have been reported at $6-20 \% .{ }^{5,6,8,9}$

\section{ANSWER 5}

Complex reconstructions are contraindicated initially due to the possibility of requiring re-excision. In the earlobe, wedge excisions provide good tumour clearance, avoid gross distortion and have good aesthetic results. ${ }^{10}$ However, through the pinna, wedges have significant risk of dehiscence and disortion. ${ }^{10}$

No consensus exists regarding optimal surgical management of ear melanoma. Common strategies include direct closure, flaps, grafts and wedge excisions. ${ }^{11}$

\section{Key points}

- Patients may be unaware of their skin malignancies.

- Recurrence can occur after two years postoperatively and new primary skin malignancies can arise; therefore, long-term follow up of high-risk individuals is crucial.

- Adequate and representative biopsy sample is critical.

- If discordance between clinical and histological findings exists, liaison with dermatology and, if indicated, re-biopsy is critical.

- Facial surgery can be done without significant disfigurement, but complex repairs should be avoided before confirmation of clear margins. 


\section{Authors}

Sarah Morton MBBS, Resident Medical Officer, Royal Brisbane and Women's Hospital, Qld

Jim Muir MBBS, FACD, FACRRM (Hon), Consultant

Dermatologist, Mater Hospital, Qld; Associate

Professor, University of Queensland, Qld

Daniel Kennedy MBBS, FRACS (Plast), President,

Australian Society of Plastic Surgeons; Plastic

Surgeon, Tele-Derm National, NSW

Competing interests: None.

Funding: None.

Provenance and peer review: Not commissioned, externally peer reviewed.

\section{Correspondence to:}

sarah.morton@health.qld.gov.au

\section{Acknowledgements}

The authors would like to thank Dr Louis Pool, Dermatopathologist, Sullivan Nicolaides Pathology, for provision of the histopathology images and commentary.

\section{References}

1. Star P, Guitera P. Lentigo maligna, macules of the face, and lesions on sun-damaged skin confocal makes the difference. Dermatol Clin 2016;34(4):421-29. doi: 10.1016/j.det.2016.05.005.

2. Akhtar S, Bhat W, Magdum A, Stanley PR. Surgical excision margins for melanoma in situ. J Plast Reconstr Aesthet Surg 2014;67(3):320-23. doi: 10.1016/j.bjps.2013.11.014

3. Byrom L, Barksdale S, Weedon D, Muir J. Unstable solar lentigo: A defined separate entity. Australas J Dermatol 2016;57(3):229-34. doi: 10.1111/ajd.12447.

4. Dalton SR, Gardner TL, Libow LE, Elston DM. Contiguous lesions in lentigo maligna. J Am Acad Dermatol 2005;52(2):859-62. doi: 10.1016/j. jaad.2004.11.063.

5. Cancer Council Australia. Cancer Council Australia Melanoma Guidelines Working Party. Clinical practice guidelines for the diagnosis and management of melanoma. Sydney, NSW: Cancer Council Australia, 2021. Available at https://wiki. cancer.org.au/australia/Guidelines:Melanoma [Accessed 19 February 2021].

6. Kunishige JH, Doan L, Brodland DG, Zitelli JA. Comparison of surgical margins for lentigo maligna versus melanoma in situ. J Am Acad Dermatol 2019;81(1):204-12. doi: 10.1016/j. jaad.2019.01.051.

7. National Institute for Healthcare and Excellence. Melanoma: Assessment and management. London, UK: NICE, 2015.

8. Connolly KL, Hibler BP, Lee EH, Rossi AM, Busam KJ, Nehal KS. Locally recurrent lentigo maligna and lentigo maligna melanoma: Characteristics and time to recurrence after surgery. Dermatol Surg 2017;43(6):792-97. doi: 10.1097/DSS.0000000000001118.

9. Fröhlich SM, Cazzaniga S, Kaufmann LS Hunger RE, Seyed Jafari SM. A retrospective cohort study on patients with lentigo maligna melanoma. Dermatology 2019;235(4):340-45. doi: 10.1159/000499689.

10. Lykoudis EG, Seretis K, Demiri E. Auricular wedge excision revisited: Technical refinements to avoid postoperative ear deformities. Plast Reconstr Surg 2013;131(5):853e-54e. doi: 10.1097/ PRS.0b013e318287a10a.

11. Toia F, Garbo G, Tripoli M, Rinaldi G, Moschella F, Cordova A. A systematic review on external ear melanoma. J Plast Reconstr Aesthet Surg 2015;68(7):883-94. doi: 10.1016/j. bjps.2015.04.003. 